\section{Understanding the neurobiology of emotion perception: implications for psychiatry}

\author{
M. L. PHILLIPS
}

Intact perception and experience of emotion is vital for survival in the social environment. In recent years, there has been increasing interest in the examination of the neurobiological basis of emotion perception in humans and non-human primates, and an acknowledgement that abnormalities in emotion perception may be associated with specific symptoms of psychiatric disorders, including schizophrenia and affective disorders (Borod \& Madigan, 2000). In this editorial, a brief outline will be presented of findings from studies employing a variety of techniques, which have helped to increase understanding of the neurobiological processes underlying normal emotion perception. Findings from other studies will then be described, which have examined the associations between specific abnormalities in these processes and symptoms of schizophrenia, bipolar and major depressive disorders.

\section{THE NEUROBIOLOGICAL BASIS OF NORMAL EMOTION PERCEPTION}

Emotion perception can be understood in terms of three related processes: (a) the identification of emotionally salient information in the environment; (b) the generation of emotional experiences and behaviour in response to (a); (c) the regulation of emotional experiences and behaviour. The last may involve an inhibition of processes (a) and (b) so that the affective state and behaviour generated in response to environmental stimuli are contextually appropriate. Findings from studies which have increased understanding of the nature of the neural correlates of these processes, in particular processes (a) and (b), are outlined below.

\section{The identification of emotionally salient information}

A well-described 'limbic' circuit, including ventral regions of the anterior cingulate gyrus, the ventromedial prefrontal cortex, the ventral striatum and the dorsomedial nucleus of the thalamus, has been previously identified as potentially important for motivation and emotion-processing (Alexander et al, 1990). Specific neural regions also have been highlighted as particularly important for the identification of emotional stimuli, and include the amygdala and anterior insula (Calder et al, 2001).

The amygdala is a small, almondshaped region within the anterior part of the temporal lobe. Several studies of non-human primates and of patients with amygdala lesions, and studies employing functional neuroimaging techniques, have demonstrated the importance of the amygdala in face and eye-gaze identification (Davis \& Whalen, 2001). Other studies have emphasised the importance of the amygdala for the identification of emotional expressions displayed by others, in particular threat-related emotions such as fear, but also sadness and happiness. In addition, the amygdala is involved in the response to non-facial displays of emotion, including unpleasant auditory, olfactory and gustatory stimuli, and in memory for emotional information (Calder et al, 2001).

The insula is a part of the cerebral cortex at the base of the lateral fissure. Studies have demonstrated the role of the anterior (agranular) insula, in particular in the autonomic response to aversive stimuli, fear reactivity and anticipatory anxiety, and in the identification of displays of disgust in others (Phillips et al, 1997; Calder et al, 2001).

\section{The generation of emotional experiences and behaviours}

Studies of non-human primates, of patients with amygdala lesions and of patients with temporal lobe epilepsy, in whom abnormal activation of the amygdala occurs, have demonstrated the role of the amygdala in the generation of appropriate emotional experiences and behaviours in response to emotionally salient, particularly fearful, stimuli (Calder et al, 2001). Other studies using similar techniques have highlighted the role of the insula, particularly the anterior insula, in the generation of the response to aversive and disgust-provoking material. For example, the insula has been shown to be important for conditioned taste aversion, unpleasant taste, pain perception, anxiety and nausea (Calder et al, 2001).

Other regions involved in the response to emotionally salient material include the ventral region of the anterior cingulate gyrus and ventromedial and ventrolateral regions of the prefrontal cortex. The ventral anterior cingulate gyrus, including subgenual and pregenual or rostral regions, is important for autonomic function and emotional behaviour. Studies employing functional neuroimaging techniques in humans have demonstrated activity within this structure in mood induction and during anxiety associated with the anticipation of pain (Drevets, 2000). The ventromedial prefrontal cortex and the medial region of this structure, the orbitofrontal cortex which has direct connections with the amygdala, appear to be particularly important in animals and humans for: the perception of pleasant and unpleasant odours, flavours and tactile stimuli; the representation of the reward value of a stimulus; and the way in which this representation then guides goal-directed and normal social behaviour (Damasio, 1994). The ventrolateral prefrontal cortex lies lateral to the orbitofrontal cortex on the ventral surface of the frontal lobes. Human functional neuroimaging studies have demonstrated activity within this region during the response to emotional information, including the induction of sad mood and the recall of personal memories and emotional material (Drevets, 2000).

\section{The regulation of emotional experiences and behaviour}

To date, there is limited understanding of the neural basis of emotion regulation. There is some indication, primarily from studies employing functional neuroimaging techniques in humans, for the role in this process of a dorsal neural system. This would comprise dorsal regions of the anterior cingulate gyrus and dorsomedial and dorsolateral prefrontal cortices, structures which are important for selective attention, planning, motor responses to emotional 
stimuli and the integration of these processes with emotional input (Drevets, 2000).

Overall, these findings indicate the involvement in the perception of emotionally salient information of a distributed neural system which includes the ventral striatum, specific thalamic nuclei, the amygdala, the anterior insula and ventromedial regions of the prefrontal cortex. Whereas the ventral striatum, dorsomedial nucleus of the thalamus, amygdala and anterior insula appear to be important for the identification of emotionally salient stimuli, the ventromedial and ventrolateral prefrontal cortical regions appear to be of particular importance for the generation of emotional experiences and behaviour in response to these stimuli. Although the neural correlates of emotion regulation remain to be elucidated, dorsal prefrontal regions may have a particular role in this process (Fig. 1).

\section{IMPLICATIONS FOR UNDERSTANDING SYMPTOMS OF PSYCHIATRIC DISORDERS}

Several studies have provided evidence for the presence of specific abnormalities in neural regions important for the identification of emotional material and the generation of emotional responses in patients with major psychiatric disorders, including schizophrenia, bipolar and major depressive disorders.

\section{Schizophrenia}

Abnormalities in emotional expression identification and emotional behaviour have been demonstrated in patients with schizophrenia in several studies, and have been associated with the poor social function and dysfunctional emotional experience demonstrated in these patients (Borod \& Madigan, 2000). Neuropathological and structural neuroimaging studies of these patients have demonstrated abnormal neuronal cell integrity and volume reductions in the amygdala, insula, thalamus and hippocampus (Wright et al, 2000); studies employing functional neuroimaging techniques have demonstrated a failure to activate limbic regions in response to emotive stimuli (Crespo-Facorro et al, 2001). The effect of medication upon these structural and functional neural abnormalities remains unclear.

\section{Bipolar disorder}

In patients with bipolar disorder, there have been reports of impaired recognition of happy and sad facial expressions, and a bias

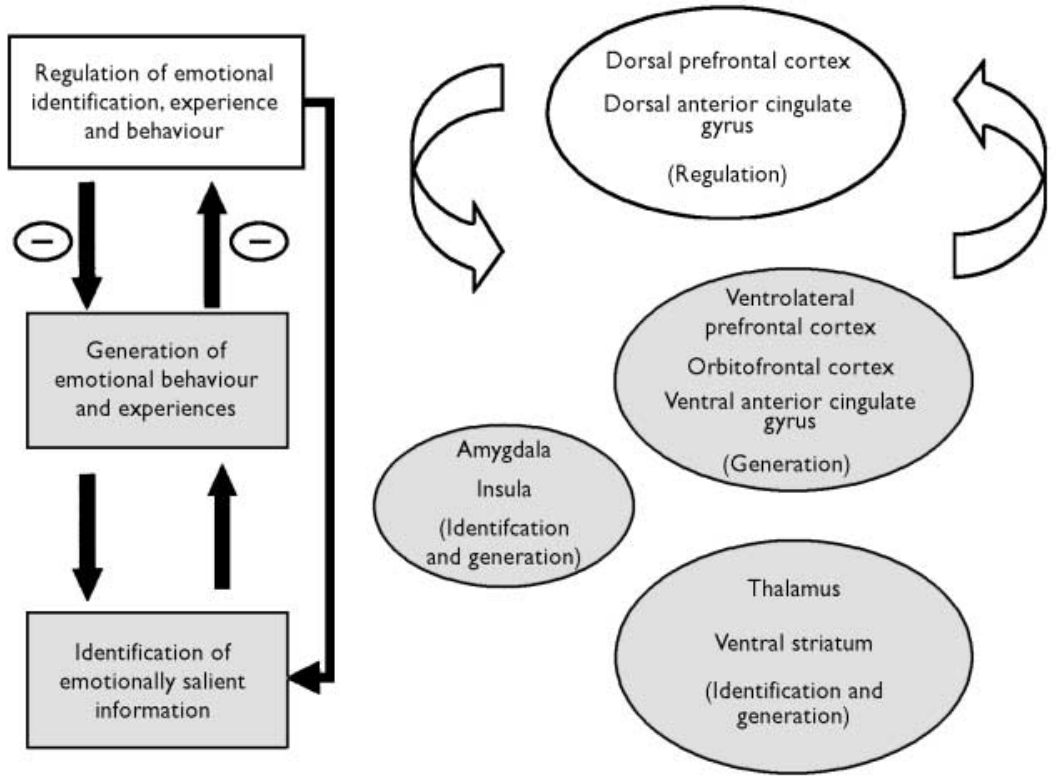

Fig. I The left-hand diagram depicts the relationships of the three main processes important for emotion perception. The right-hand diagram depicts neural structures important for these three processes, and their putative functional relationships.

towards the recognition of sad facial expressions (Gur et al, 1992). Major findings from neuropathological and structural neuroimaging studies in patients with bipolar disorder include reduced glial cell number and density and volume reductions within the ventral anterior cingulate gyrus (specifically, the subgenual region of the ventral anterior cingulate gyrus), and increases in amygdalar volumes. However, there are inconsistent findings regarding structural changes within other limbic regions (Stoll et al, 2000). These studies have been unable to distinguish between abnormalities caused by and/or associated with the depressive, euthymic and manic phases of the disorder.

Studies employing functional neuroimaging techniques have demonstrated: increased blood flow within the subgenual region of the ventral anterior cingulate gyrus during sad-mood induction in patients with bipolar disorder; decreased activity within this region in patients with bipolar depression; and increased activity in this region in patients with bipolar mania during word-generation and at rest (Drevets, 2000). Within the amygdala, studies have demonstrated increased activation in response to facial expressions of fear and, in patients with bipolar depression, increased metabolism during performance of an auditory continuous performance test (Drevets, 2000; YurgelunTodd et al, 2000). There are other reports of decreased cerebral blood flow and activity within the ventromedial prefrontal (orbitofrontal) cortex during wordgeneration and at rest in manic compared with euthymic phases, and during a decision-making task in mania compared with depression (Blumberg et al, 1999; Rubinsztein et al, 2001).

\section{Major depressive disorder}

In patients with major depressive disorder, studies have demonstrated either generalised or specific impairments in the identification of emotional facial expressions, or a bias towards the identification of expressions as sad (Gur et al, 1992). Neuropathological and structural neuroimaging studies in patients with major depressive disorder have indicated abnormalities within the subgenual anterior cingulate gyrus similar to those in patients with bipolar disorder, and volume reductions within the hippocampus, ventral striatal regions and amygdala. However, there have been 
discrepant findings, possibly owing to differences in medication status, acquisition paradigms and image resolution (Drevets, 2000). Studies employing functional neuroimaging techniques have demonstrated in patients during a major depressive episode increased metabolism and blood flow within the subgenual cingulate gyrus, if the reduction in subgenual cingulate gyral volumes in these patients is corrected (Mayberg et al, 2000), and increased activity within the amygdala (Sheline et al, 2001). Improvement in symptoms has been associated with reduced metabolism in the ventral/subgenual cingulate gyrus and insula (Mayberg et al, 2000).

Taken together, these findings suggest that specific abnormalities in the structure and function of neural regions important for the identification of and response to emotional material may be associated with social dysfunction in schizophrenia, emotional lability in bipolar disorder and the negative bias and depressed mood of major depressive disorder.

\section{CONCLUSIONS}

Findings from studies employing a variety of techniques have highlighted the importance of the amygdala, ventral striatum and other limbic regions, together with ventral prefrontal cortical regions, in the identification of and response to emotionally salient information in the environment. Specific abnormalities both in the identification of and response to emotional material are associated with symptoms of a variety of psychiatric disorders, including schizophrenia, bipolar and major depressive

M. L. PHILLIPS, MRCPsych, Institute of Psychiatry, De Crespigny Park, London SE5 8AF, UK. Tel: +44 (0)20 7848 0379; fax: +44 (0)20 7848 0379; e-mail: spmamlp@iop.kcl.ac.uk

(First received 28 March 2002, final revision 5 June 2002, accepted 5 June 2002)

disorders. Structural and functional abnormalities within many of the neural regions important for these processes are present in these psychiatric populations. These findings suggest that increased knowledge of the neurobiology of normal emotion perception will help to further understanding of the complex relationship between specific abnormalities of emotion perception, related deficits in neural systems important for emotion perception, and the symptoms of a wide range of psychiatric disorders.

\section{REFERENCES}

Alexander, G. E., Crutcher, M. D. \& DeLong, M. R. (1990) Basal ganglia-thalamocortical circuits: parallel substrates for motor, oculomotor, "prefrontal" and "limbic" functions. Progress in Brain Research, 85, 119-146.

Blumberg, H. P., Stern, E., Ricketts, S., et al (1999) Rostral and orbital prefrontal cortex dysfunction in the manic state of bipolar disorder. American Journal of Psychiatry, 156, 1986-1988.

Borod, J. C. \& Madigan, N. K. (2000)

Neuropsychology of emotion and emotional disorders: an overview and research directions. In The

Neuropsychology of Emotion (ed. J. C. Borod), pp. 3-28. New York: Oxford University Press.

Calder, A. J., Lawrence, A. D. \& Young, A.W. (200I) Neuropsychology of fear and loathing. Nature Reviews Neuroscience, 2, 352-363.

Crespo-Facorro, B., Paradiso, S., Andreasen, N. C., et al (200I) Neural mechanisms of anhedonia in schizophrenia. JAMA, 286, 427-435.
Damasio, A. R. (1994) Descartes' error and the future of human life. Scientific American, 27I, 144

Davis, M. \& Whalen, P. J. (200I) The amygdala: vigilance and emotion. Molecular Psychiatry, 6, 13-34

Drevets, W. C. (2000) Neuroimaging studies of mood disorders. Biological Psychiatry, 48, 813-829.

Gur, R. C., Erwin, R. J., Gur, R. E., et al (1992) Facial emotion discrimination. II: Behavioral findings in depression. Psychiatric Research, 42, 24I-25|.

Mayberg, H. S., Brannan, S. K., Tekell, J. L., et al (2000) Regional metabolic effects of fluoxetine in major depression: serial changes and relationship to clinical response. Biological Psychiatry, 48, 830-843.

Phillips, M. L., Young, A.W., Senior, C., et al (1997) A specific neural substrate for perception of facial expressions of disgust. Nature, 389, 495-498.

Rubinsztein, J. S., Fletcher, P. C., Rogers, R. D., et al (200I) Decision-making in mania: a PETstudy. Brain, 124 2550-2563.

Sheline, Y. I., Barch, D. M., Donnelly, J. M., et a (200I) Increased amygdalar response to masked emotional faces in depressed subjects resolves with antidepressant treatment: an fMRI study. Biological Psychiatry, 50, 65I-659.

Stoll, A. L., Renshaw, P. F., Yurgelun-Todd, D. A., et a (2000) Neuroimaging in bipolar disorder: what have we learned? Biological Psychiatry, 48, 505-517.

Wright, I. C., Rabe-Hesketh, S., Woodruff, P. W. R., et al (2000) Regional brain structure in schizophrenia: a meta-analysis of volumetric MRI studies. American Journal of Psychiatry, 157, 16-25.

Yurgelun-Todd, D. A., Gruber, S. A., Kanayama, G., et al (2000) fMRI during affect discrimination in bipolar affective disorder. Bipolar Disorders, 2, 248. 